\title{
Multitarget tracking in cluttered environment for a multistatic passive radar system under the DAB/DVB network
}

\author{
Yi Fang Shi, Seung Hyo Park and Taek Lyul Song*
}

\begin{abstract}
The target tracking using multistatic passive radar in a digital audio/video broadcast (DAB/DVB) network with illuminators of opportunity faces two main challenges: the first challenge is that one has to solve the measurement-to-illuminator association ambiguity in addition to the conventional association ambiguity between the measurements and targets, which introduces a significantly complex three-dimensional (3-D) data association problem among the target-measurement illuminator, this is because all the illuminators transmit the same carrier frequency signals and signals transmitted by different illuminators but reflected via the same target become indistinguishable; the other challenge is that only the bistatic range and range-rate measurements are available while the angle information is unavailable or of very poor quality.

In this paper, the authors propose a new target tracking algorithm directly in three-dimensional (3-D) Cartesian coordinates with the capability of track management using the probability of target existence as a track quality measure. The proposed algorithm is termed sequential processing-joint integrated probabilistic data association (SP-JIPDA), which applies the modified sequential processing technique to resolve the additional association ambiguity between measurements and illuminators. The SP-JIPDA algorithm sequentially operates the JIPDA tracker to update each track for each illuminator with all the measurements in the common measurement set at each time. For reasons of fair comparison, the existing modified joint probabilistic data association (MJPDA) algorithm that addresses the 3-D data association problem via "supertargets" using gate grouping and provides tracks directly in 3-D Cartesian coordinates, is enhanced by incorporating the probability of target existence as an effective track quality measure for track management. Both algorithms deal with nonlinear observations using the extended Kalman filtering. A simulation study is performed to verify the superiority of the proposed SP-JIPDA algorithm over the MJIPDA in this multistatic passive radar system.
\end{abstract}

Keywords: Multistatic passive radar, DAB/DVB, MJIPDA, SP-JIPDA, Track management

\section{Introduction}

In a multistatic passive radar system, illuminators of opportunity such as radio or television transmitters can be used. The transmitted signals are not under the control of the receiver; thus, the receiver can remain hidden. One can measure the time difference of arrival (TDOA) and Doppler shift between signal received directly from the illuminator and delayed copies from potential targets. As the receiver and transmitter in the multistatic passive

${ }^{*}$ Correspondence: tsong@hanyang.ac.kr

Department of Electronic Systems Engineering, Hanyang University, 55

Hanyangdaehak- ro, Ansan, Republic of Korea radar system are completely separated, the receiver therein just needs to process the received signals while it has to service the transmitter in a feedback manner and consumes much more power in some other radar systems; therefore, the multistatic passive radar system is more economic. Due to the non-cooperative illuminator transmits low RF signals, the chance to detect stealth and low altitude targets increases [1]; however, this causes problem in that measurement of the azimuth angle is often of very poor quality or not even available at some extreme situations. Therefore, in this multistatic passive radar system, the range and range rate are measured while the angle 
information is assumed to be unavailable, due to the low RF frequencies of the illuminating signals.

In this paper, we focus on target tracking from preprocessed detections originating from the television or radio broadcasting signals that are modulated according to the digital audio broadcasting (DAB) or digital video broadcasting (DVB) standards $[2,3]$. The using of the DAB/DVB signals delivers numbers of advantages compared to that of analog signals: such as, the improved detection performance [4], the more effective signal processing process [5], and the more easily estimated multipath. As a result, a couple of widely spaced transmitters that broadcast the $\mathrm{DAB} / \mathrm{DVB}$ signals on the same carrier frequency with each responsible for a small and overlapping subscriber footprint, are used to cover a large surveillance space.

However, there are two main challenges in this multistatic passive radar system with a DAB/DVB network. One is that there is a new association ambiguity between the measurements and the illuminators on top of the conventional ambiguity between the measurements and targets, which results in three-dimensional (3-D) data association and adds significant data association complexity. This is because all illuminators in this system transmit the same frequency broadcasting signals and it is not available for the receiver to differentiate the received signals from the different illuminators' signals. The other challenge is that more than one illuminator is needed to locate a single target due to the absence of angle information, which inevitably generates a lot of ghosts.

There has been numbers of research focused on the problems in this multistatic passive radar system with DAB/DVB networks. The track-before-detect (TBD) algorithms $[6,7]$ that estimate the target positions directly from the unprocessed DAB/DVB signals can be used to solve the association measurements since the target is observed over several consecutive scans and hence reducing the number of possible associations. The TBD algorithms ensure better detection and estimation performance than conventional algorithms at the price of an increased computational load, while the empirical techniques can be adopted to reduce the complexity of the TBD algorithms, one can refer to [8-10], therein, the proposed algorithms have a complexity linear in the number of integrated scans and in the time on target. As opposed to from the unprocessed signals, most other researchers focus on the target position estimation from the preprocessed detections (measurements), which require less computation source over the TBD algorithms but have to address the data association problem. The authors in [11] propose a multi-dimensional assignment approach to solve the transmitter ambiguity for bistatic range, range rate, and precise azimuth measurements. In [12], the authors propose a multi-hypothesis tracking (MHT)based three-stage approach that includes primary tracking directly on the measurements and a two-dimensional (2-D) estimate to address the association problem that is later resolved into 3-D. Another algorithm employing the likelihood ratio test to remove "ghost" tracks is investigated in [13]. Recently, Choi et al. [14, 15] propose two groups of algorithms for multitarget tracking directly in the Cartesian coordinates. One group consists of the extended Kalman filter (EKF) and unscented Kalman fitler (UKF) [16] based modified joint probabilistic data association (MJPDA) to resolve the additional ambiguity between measurements and illuminators, while the other group consists of the bootstrap particle filter (BPF) and auxiliary particle filter (APF) [17] based data association under the probabilistic multi-hypothesis tracker (PMHT) measurement model. However, these preprocessed-detection-based tracking algorithms neglect providing an effective track quality measure for track management, which motivates the authors to consider the probability of target existence as a track quality measure for multitarget tracking from preprocessed detections.

This paper focuses on the multitarget tracking algorithms directly in three-dimensional (3-D) Cartesian coordinates using the preprocessed detections. Motivated by the techniques presented in $[18]$ and $[19,20]$. The authors propose a new algorithm entitled sequential processing-joint integrated probabilistic data association (SP-JIPDA) which provides tracks directly in 3-D Cartesian coordinates, and enable the track management using the probability of target existence as a track quality measure. To the best knowledge of the authors, the only existing multitarget tracking algorithms directly in 3D Cartesian coordinates under the multistatic DAB/DVB passive radar system are proposed in $[14,15]$, they are the modified joint probabilistic data association (MJPDA) and the particle filtering under the probabilistic multiple hypothesis (PMHT) model, with the MJPDA delivers more robust tracking performance and requires less computational resources. However, both of the exiting algorithms neglect providing an efficient track quality measure for track management. Therefore, in order to compare the proposed algorithm (SP-JIPDA) to the exiting algorithms, the MJPDA algorithm is enhanced by incorporating the probability of target existence as a track quality measure for track management, and termed modified joint integrated probabilistic data association (MJIPDA). The proposed SP-JIPDA algorithm avoids the extra data association ambiguity between the measurements and illuminators, and moreover, the measurement information from various illuminators is utilized in a more effective way and delivers much better tracking performance compared with the MJIPDA algorithm.

The paper is organized as follows. Section 2 describes the problem statement, and the modified JIPDA algorithm 
is presented in Section 3. Section 4 investigates the sequential processing JIPDA algorithm and the simulation is implemented in Section 5, with the conclusions in Section 6.

\section{Problem statement}

The tracking algorithms presented in this paper are based on the infinite sensor resolution and point target assumptions. Denote a track or a potential target followed by a certain track by superscript $\tau$, of which the interpretation is clear from the context.

\subsection{Target}

Let $\chi_{k}^{\tau}$ denote the random existence event for a target being followed by track $\tau$ at time $k$. The event $\chi_{k}^{\tau}$ propagates as a Markov process [21-23]. Denote the trajectory state with position and velocity components in 3-D Cartesian coordinates of target $\tau$ at time $k$ by $\mathbf{x}_{k}^{\tau}=\left[\begin{array}{ll}\mathbf{x}_{k}^{\tau, p} & \mathbf{x}_{k}^{\tau, v}\end{array}\right]^{T}$, with $\mathbf{x}_{k}^{\tau, p}=\left[\begin{array}{lll}x^{\tau}(k) & y^{\tau}(k) & z^{\tau}(k)\end{array}\right]^{T}$ and $\mathbf{x}_{k}^{\tau, v}=\left[\begin{array}{lll}\dot{x}^{\tau}(k) & \dot{y}^{\tau}(k) & \dot{z}^{\tau}(k)\end{array}\right]^{T}$. The trajectory state is assumed to propagate linearly by

$$
\mathbf{x}_{k+1}^{\tau}=\mathbf{F} \mathbf{x}_{k}^{\tau}+\mathbf{v}_{k}^{\tau}
$$

where $\mathbf{v}_{k}^{\tau}$ is the white Gaussian noise sequence with zero mean and covariance $\mathbf{Q}_{k}$, and $\mathbf{F}$ denotes the state transition matrix, which is calculated by

$$
\mathbf{F}=\left[\begin{array}{ll}
1 & T \\
0 & 1
\end{array}\right] \otimes \mathbf{I}_{3}
$$

where $T$ is the sampling interval between two consecutive scans, $\otimes$ denotes the Kronecker product and $\mathbf{I}_{3}$ is the identity matrix of size 3 . For reasons of simplicity, as well as better concentrating on the essence of problems caused by multitarget tracking in clutter for a multistatic passive radar system under the DAB/DVB network, we assume a constant velocity target trajectory model. There have two feasible methods to face with the possible target trajectory changes: one is to increase the value of target trajectory plant noise covariance matrix $\left(\mathbf{Q}_{k}\right)$ when the target trajectory changes slightly, therein, the increased plant noise covariance is able to account for certain amount of mismatch between the assumed trajectory model and the actual one. However, if the target trajectory changes dramatically or even maneuvers among different motion models, one can resort to the interactive multiple model (IMM) algorithm [24], which performs well on systems characterized by multiple models of target behavior.

Each existing target $\tau$ at time $k$ creates at most one detection with probability of detection $P_{D}$. In this multistatic passive radar system of DAB/DVB network, there exist multiple illuminators located at $\mathbf{x}_{s}=\left[\begin{array}{lll}x_{s} & y_{s} & z_{s}\end{array}\right]^{T}$ for $s=1, \cdots, N_{s}$ and only one receiver located at $\mathbf{x}_{r}=\left[\begin{array}{lll}x_{r} & y_{r} & z_{r}\end{array}\right]^{T}$, the receiver can measure bistatic range $\gamma_{k}$ and range rate $\dot{\gamma}_{k}$ at time $k$, which are given by

$$
\gamma_{k}\left(\mathbf{x}_{k}^{\tau}, \mathbf{x}_{s}\right)=\left\|\mathbf{x}_{k}^{\tau, p}-\mathbf{x}_{r}\right\|+\left\|\mathbf{x}_{k}^{\tau, p}-\mathbf{x}_{s}\right\|,
$$

$$
\dot{\gamma}_{k}\left(\mathbf{x}_{k}^{\tau}, \mathbf{x}_{s}\right)=\frac{\left(\mathbf{x}_{k}^{\tau, p}-\mathbf{x}_{r}\right)^{T} \mathbf{x}_{k}^{\tau, v}}{\left\|\mathbf{x}_{k}^{\tau, p}-\mathbf{x}_{r}\right\|}+\frac{\left(\mathbf{x}_{k}^{\tau, p}-\mathbf{x}_{s}\right)^{T} \mathbf{x}_{k}^{\tau, v}}{\left\|\mathbf{x}_{k}^{\tau, p}-\mathbf{x}_{s}\right\|}
$$

Thus, the observation with respect to illuminator $s$ is given as

$$
\mathbf{z}_{k}=h_{k}^{s}\left(\mathbf{x}_{k}^{\tau}, \mathbf{x}_{s}\right)+\mathbf{w}_{k},
$$

where $\mathbf{z}_{k}=\left[\gamma_{k} \dot{\gamma}_{k}\right]^{T}, h_{k}^{s}\left(\mathbf{x}_{k}^{\tau}, \mathbf{x}_{s}\right)=\left[\gamma_{k}\left(\mathbf{x}_{k}^{\tau}, \mathbf{x}_{s}\right) \dot{\gamma}_{k}\left(\mathbf{x}_{k}^{\tau}, \mathbf{x}_{s}\right)\right]^{T}$, and $\mathbf{w}_{k}$ is the white Gaussian noise sequence with zero mean and covariance $\mathbf{R}_{k}$, uncorrelated with the plant noise sequence $\mathbf{v}_{k}^{\tau}$. At each time $k$, the receiver receives a random set of measurements $\mathbf{Z}_{k}$ without prior information on the origin of each measurement. Each measurement has only one resource, either a target or clutter, but it can be originated from any illuminator. Denote the set of measurements up to and including time $k$ by $\mathbf{Z}^{k}=$ $\left\{\mathbf{Z}^{k-1}, \mathbf{Z}_{k}\right\}$, and let $\mathbf{Z}_{k, i}$ denote the $i$ th measurement of $\mathbf{Z}_{k}$.

\subsection{Clutter}

At each scan, the sensor received a random number of clutter (false) measurements. The number of clutter measurements in the surveillance space is usually modeled by a Poisson distribution [24] with somehow known intensity, which is termed as the clutter measurement density. Denote the clutter measurement density of measurement $\mathbf{Z}_{k, i}$ by the shortcut $\rho_{k, i} \triangleq \rho\left(\mathbf{Z}_{k, i}\right)$. Assume the volume of the surveillance space at time $k$ is $V_{k}$, thus the probability that the number of the clutter measurements equals $m$ in $V_{k}$ at time $k$ follows the Poisson distribution

$$
u_{F}(m)=e^{-\int_{V_{k}} \rho_{k, i} d V} \frac{\left(\int_{V_{k}} \rho_{k, i} d V\right)^{m}}{m !},
$$

where the clutter measurement density $\rho_{k, i}=\frac{P_{f a}}{V_{r c}}[24,25]$, with $P_{f a}$ and $V_{r c}$ denote the probability of false alarm and the sensor resolution cell volume. As can be easily seen, the probability of false alarm impacts on the number of clutter measurements significantly, that is, if the probability of false alarm $P_{f a}$ increases, the clutter measurement density $\rho_{k, i}$ also increases, and the mean number of clutter measurements in the surveillance space $V_{k}$ increases, which results in a increased number of clutter measurements at each time $k$. In target tracking, the clutter measurement density is either a priori known or estimated adaptively based on the current measurements [26]. 


\subsection{Multistatic passive radar in a DAB/DVB network}

As the focus of this paper is on target tracking from detections of the preprocessed signals in the multistatic passive radar systems of a DAB/DVB network, there mainly exist two challenges. One is that there is a new association ambiguity between measurements and illuminators besides the conventional one between measurement and targets, which results in 3-D data association among target-measurement illuminators. This is because each of the multiple illuminators transmits the same frequency digital signal, albeit with different delays due to the illuminator-target-receiver geometry, the fact that received signals are composed of multiple unlabeled delays per target, and that there is no useful information to discriminate the origin of the measurement. As the number of 3-D association events increases dramatically [15] even given small number of measurements, targets and illuminators, it is computationally unrealistic to directly implement conventional 2-D data association approaches to resolve the 3-D data association problem in this passive radar system.

The other challenge is due to the broadcasting signal frequencies for passive radars and the type of receivers, the angle information is of realistically poor quality and target tracking using only the range and range rate without angle information inevitably generates ghost tracks. A bistatic range measurement can locate a target at an ellipsoid in 3-D Cartesian coordinates, but for the intersection of two ellipsoids, which is an ellipse, a third bistatic range measurement is necessary to possibly locate the target, resulting in the generation of multiple ghosts. Furthermore, in the presence of measurement noise and the clutter measurement, the situation is even more severe.

In the following two sections, two track maintenance algorithms directly in 3-D Cartesian coordinates are introduced. The first presented in Section 3 is the modified JIPDA algorithm, which is enhanced by incorporating the probability of target existence as a track quality measure. The second algorithm, proposed in Section 4 is the sequential processing JIPDA algorithm, which is our main contribution of this paper.

\section{Modified JIPDA (MJIPDA)}

\subsection{Supertarget and gate grouping}

The JPDA algorithm [24] enumerates and probabilistically evaluates every validated measurement to the target association event, and the target states are estimated by using the marginal association probability. In this multistatic passive radar system, as discussed in Section 2.3, the computational cost of evaluating all possible 3-D association events is much higher than the cost for 2-D association events. The authors in [15] proposed a suboptimal idea of supertarget $\tilde{\tau}=\{\tau, s\}$, which is a hypothetical target consisting of a pair of target $\tau$ and illuminator $s$, and succeeded to recast the 3-D association among measurements, targets and illuminators to a 2-D list-matching problem between measurements and supertargets.

Given that the number of association events increases in the number of targets (supertargets) and measurements involved, the gate grouping concept is introduced [27]. Denote the set of gating validated measurements at time $k$ with respect to supertarget $\tilde{\tau}$ by $\mathbf{z}_{k}^{\tilde{\tau}}$, with corresponding set cardinality $N_{k}^{\tilde{\tau}} \cdot \mathbf{z}_{k, i}^{\tilde{\tau}}$ is the $i$ th measurement of $\mathbf{z}_{k}^{\tilde{\tau}}$.

$$
\begin{array}{r}
\mathbf{z}_{k}^{\tilde{\tau}}=\left\{\mathbf{Z}_{k, i} \in \mathbf{Z}_{k}:\left(\mathbf{Z}_{k, i}-h_{k}^{s}\left(\mathbf{x}_{k \mid k-1}^{\tau}, \mathbf{x}_{s}\right)\right)^{T}\left(\mathbf{S}_{k}^{\tau, s}\right)^{-1}\right. \\
\left.\left(\mathbf{Z}_{k, i}-h_{k}^{s}\left(\mathbf{x}_{k \mid k-1}^{\tau}, \mathbf{x}_{s}\right)\right)<\kappa\right\}
\end{array}
$$

with the predicted measurement $h_{k}^{s}\left(\mathbf{x}_{k \mid k-1}^{\tau}, \mathbf{x}_{s}\right)$ and its associated covariance $\mathbf{S}_{k}^{\tau, s}$ with respect to supertarget $\tilde{\tau}=$ $\{\tau, s\}$, where $\kappa$ is the gating threshold.

The supertargets sharing at least one measurement in their corresponding validation gates are classified as one group. Thus, all of the 3-D association events can be separated as groups of 2-D association events, which decreases the number of association events on a large scale. Note that the concept of a group here is slightly different from the cluster, in the sense that the supertargets in different groups are possibly from the same target since one target can create $N_{s}$ supertargets via combining with $N_{s}$ illuminators.

Table 1 gives an example of four supertargets, which are formed by combinating two targets $\left(\tau_{1}, \tau_{2}\right)$ and two illuminators $\left(s_{1}, s_{2}\right)$, respectively, as well as their corresponding validated measurements. There are two groups: the first group is $\left(\tilde{\tau}_{1}, \tilde{\tau}_{3}\right)$ with shared validated measurements $\left(\mathbf{z}_{2}, \mathbf{z}_{4}\right)$, the other group is $\left(\tilde{\tau}_{2}, \tilde{\tau}_{4}\right)$ with shared validated measurements $\left(\mathbf{z}_{1}, \mathbf{z}_{3}\right)$.

\subsection{Modified JIPDA using supertargets}

The proposed modified JPDA algorithm in [15] declares a track lost if no measurement falls within the target gates for some consecutive scans or the gate becomes too large. In this section, the modified JPDA is enhanced by incorporating the probability of target existence as a track quality measure for track management, which utilizes the probability of target existence to confirm or terminate

Table 1 Example of supertargets

\begin{tabular}{lllll}
\hline & $\tilde{\tau}_{1}=\left(\tau_{1}, s_{1}\right)$ & $\tilde{\tau}_{2}=\left(\tau_{1}, s_{2}\right)$ & $\tilde{\tau}_{3}=\left(\tau_{2}, s_{1}\right)$ & $\tilde{\tau}_{4}=\left(\tau_{2}, s_{2}\right)$ \\
\hline $\mathbf{z}_{1}$ & $\times$ & $\checkmark$ & $\times$ & $\checkmark$ \\
$\mathbf{z}_{2}$ & $\checkmark$ & $\times$ & $\checkmark$ & $\times$ \\
$\mathbf{z}_{3}$ & $\times$ & $\checkmark$ & $\times$ & $\checkmark$ \\
$\mathbf{z}_{4}$ & $\checkmark$ & $\times$ & $\checkmark$ & $\times$ \\
\hline
\end{tabular}


tracks, and is termed as modified joint integrated probabilistic data association (MJIPDA). The MJIPDA algorithm recursively calculates the hybrid track state at each scan. Let us start the recursion from the predicted track state of $\tau$ at time $k-1$, which consists of track existence event $\chi_{k}^{\tau}$ (discrete event) and the trajectory state $\mathbf{x}_{k}^{\tau}$ (continuous variable),

$$
p\left(\chi_{k}^{\tau}, \mathbf{x}_{k}^{\tau} \mid \mathbf{Z}^{k-1}\right)=P\left(\chi_{k}^{\tau} \mid \mathbf{Z}^{k-1}\right) p\left(\mathbf{x}_{k}^{\tau} \mid \chi_{k}^{\tau}, \mathbf{Z}^{k-1}\right)
$$

with

$$
p\left(\mathbf{x}_{k}^{\tau} \mid \chi_{k}^{\tau}, \mathbf{Z}^{k-1}\right)=\mathcal{N}\left(\mathbf{x}_{k}^{\tau} ; \hat{\mathbf{x}}_{k \mid k-1}^{\tau}, \mathbf{P}_{k \mid k-1}^{\tau}\right) .
$$

The trajectory state pdf $p\left(\mathbf{x}_{k}^{\tau} \mid \chi_{k}^{\tau}, \mathbf{Z}^{k-1}\right)$ is only conditioned on the target existence $\chi_{k}^{\tau}$, and for the rest of this paper, this conditioning is only implicit.

\subsubsection{Data association}

In this section, the authors present the multi-target data association operation on one group, where the other groups also follow the same procedure. A feasible joint event (FJE) is an allocation of all measurements to all supertargets in the group and satisfies that each supertarget is assigned zero or one measurement and each measurement is allocated to zero (clutter) or one supertarget [24]. Let $\xi_{j}$ denote the $j$ th FJE, where $T_{0}\left(\xi_{j}\right)$ and $T_{1}\left(\xi_{j}\right)$ are the set of supertargets allocated no measurement and one measurement with respect to the FJE $\xi$, respectively. The posterior probability of FJE $\xi_{j}$ is given by

$$
\begin{aligned}
P\left(\xi_{j} \mid \mathbf{Z}^{k}\right)= & c_{k} \prod_{\tilde{\tau} \in T_{0}\left(\xi_{j}\right)}\left(1-P_{D} P_{G} P\left(\chi_{k}^{\tilde{\tau}} \mid \mathbf{Z}^{k-1}\right)\right) \\
& \times \prod_{\tilde{\tau} \in T_{1}\left(\xi_{j}\right)}\left(P_{D} P_{G} P\left(\chi_{k}^{\tilde{\tau}} \mid \mathbf{Z}^{k-1}\right) \frac{p_{k, i}^{\tilde{\tau}}}{\rho_{k, i}}\right),
\end{aligned}
$$

where $P_{G}$ is the gating probability, $c_{k}$ is the normalizing constant. $P\left(\chi_{k}^{\tilde{\tau}} \mid \mathbf{Z}^{k-1}\right)=P\left(\chi_{k}^{\tau} \mid \mathbf{Z}^{k-1}\right)$ due to $\tilde{\tau}=\{\tau, s\}$.

The likelihood of measurement $\mathbf{z}_{k, i}^{\tilde{\tau}}$ allocated to supertarget $\tilde{\tau}$ in FJE $\xi_{j}$ is obtained by

$$
p_{k, i}^{\tilde{\tau}}=\frac{\mathcal{N}\left(\mathbf{z}_{k, i}^{\tilde{\tau}} ; h_{k}^{s}\left(\mathbf{x}_{k \mid k-1}^{\tau}, \mathbf{x}_{k}^{s}\right), \mathbf{S}_{k}^{\tau, s}\right)}{P_{G}},
$$

where $P_{G}$ is the gating probability. Denote by $\Xi(\tilde{\tau}, i)$ the set of FJEs which allocates measurement $i$ to supertarget $\tilde{\tau}$, and denote the event that the selected measurement $i$ is the detection of supertarget $\tilde{\tau}$ at time $k$ by $\chi_{k, i}^{\tilde{\tau}}(i=0$ means no measurement is the supertarget $\tilde{\tau}$ detection).
The posterior probability that no measurement originates from the supertarget $\tilde{\tau}$ detection is

$$
P\left(\chi_{k, 0}^{\tilde{\tau}} \mid \mathbf{Z}^{k}\right)=\sum_{\xi_{j} \in \Xi(\tilde{\tau}, 0)} P\left(\xi_{j} \mid \mathbf{Z}^{k}\right),
$$

and the posterior probability that no measurement in the group is supertarget $\tilde{\tau}$ detection as well as that supertarget $\tilde{\tau}$ exists is

$P\left(\chi_{k}^{\tilde{\tau}}, \chi_{k, 0}^{\tilde{\tau}} \mid \mathbf{Z}^{k}\right)=\frac{\left(1-P_{D} P_{G}\right) P\left(\chi_{k}^{\tilde{\tau}} \mid \mathbf{Z}^{k-1}\right)}{1-P_{D} P_{G} P\left(\chi_{k}^{\tilde{\tau}} \mid \mathbf{Z}^{k-1}\right)} P\left(\chi_{k, 0}^{\tilde{\tau}} \mid \mathbf{z}^{k}\right)$,

and the posterior probability of measurement $\mathbf{z}_{k, i}^{\tilde{\tau}}$ in the group is supertarget $\tilde{\tau}$ detection and supertarget $\tilde{\tau}$ exists is

$$
P\left(\chi_{k}^{\tilde{\tau}}, \chi_{k, i}^{\tilde{\tau}} \mid \mathbf{Z}^{k}\right)=\sum_{\xi_{j} \in \Xi(\tilde{\tau}, i)} P\left(\xi_{j} \mid \mathbf{Z}^{k}\right),
$$

thus, the posterior probability of supertarget $\tilde{\tau}$ existence is

$$
P\left(\chi_{k}^{\tilde{\tau}} \mid \mathbf{Z}^{k}\right)=\sum_{i \geq 0} P\left(\chi_{k}^{\tilde{\tau}}, \chi_{k, i}^{\tilde{\tau}} \mid \mathbf{z}^{k}\right) .
$$

The posterior data association probability for supertarget $\tilde{\tau}$ is

$$
\beta_{i \tilde{\tau}}=P\left(\chi_{k, i}^{\tilde{\tau}} \mid \chi_{k}^{\tilde{\tau}}, \mathbf{Z}^{k}\right)=\frac{P\left(\chi_{k, i}^{\tilde{\tau}}, \chi_{k}^{\tilde{\tau}} \mid \mathbf{Z}^{k}\right)}{P\left(\chi_{k}^{\tilde{\tau}} \mid \mathbf{Z}^{k}\right)}, i \geq 0 .
$$

\subsubsection{Track state update}

Since each supertarget $\tilde{\tau}$ is created by combing each track $\tau$ and each illuminator $s$ (a total of $N_{s}$ illuminators), the track state output for $\tau$ at time $k$ should involve all the supertargets $\tilde{\tau}$ originated from track $\tau$. Let $\mathrm{E}(\tau)$ denote the set of supertargets that originated from track $\tau$, with the set cardinality $N_{s}$.

$$
p\left(\chi_{k}^{\tau}, \mathbf{x}_{k}^{\tau} \mid \mathbf{Z}^{k}\right)=P\left(\chi_{k}^{\tau} \mid \mathbf{Z}^{k}\right) p\left(\mathbf{x}_{k}^{\tau} \mid \mathbf{Z}^{k}\right) .
$$

The probability density function of the track $\tau$ trajectory state is assumed to be a single Gaussian distribution,

$$
p\left(\mathbf{x}_{k}^{\tau} \mid \mathbf{Z}^{k}\right)=\mathcal{N}\left(\mathbf{x}_{k}^{\tau} ; \hat{\mathbf{x}}_{k \mid k}^{\tau}, \mathbf{P}_{k \mid k}^{\tau}\right),
$$

where

$$
\hat{\mathbf{x}}_{k \mid k}^{\tau}=\sum_{\tilde{\tau} \in \mathrm{E}(\tau)} \sum_{j=0}^{N_{k}^{\tilde{\tau}}} \tilde{c}_{k} \beta_{j j} \hat{\mathbf{x}}_{k \mid k, j}^{\tilde{\tau}}
$$

and

$$
\mathbf{P}_{k \mid k}^{\tau}=\sum_{\tilde{\tau} \in \mathrm{E}(\tau)} \sum_{j=0}^{N_{k}^{\tau}} \tilde{c}_{k} \beta_{j \tilde{\tau}}\left(\mathbf{P}_{k \mid k, j}^{\tilde{\tau}}+\hat{\mathbf{x}}_{k \mid k, j}^{\tau}\left(\hat{\mathbf{x}}_{k \mid k, j}^{\tilde{\tau}}\right)^{T}\right)-\hat{\mathbf{x}}_{k \mid k}^{\tau}\left(\hat{\mathbf{x}}_{k \mid k}^{\tau}\right)^{T} .
$$


where $\tilde{c_{k}}$ is the renormalized factor which satisfies $\sum_{\tilde{\tau} \in \mathrm{E}(\tau)} \sum_{j=0}^{N_{k}^{\tilde{\tau}}} \tilde{c_{k}} \beta_{j \tilde{\tau}}=1$. The mean and covariance updated by measurement $\mathbf{z}_{k, i}^{\tilde{\tau}}$ with respect to supertarget $\tilde{\tau}=(\tau, s)$ are calculated by

$\left[\begin{array}{ll}\hat{\mathbf{x}}_{k \mid k, i}^{\tilde{\tau}} & \mathbf{P}_{k \mid k, i}^{\tilde{\tau}}\end{array}\right]= \begin{cases}{\left[\begin{array}{ll}\hat{\mathbf{x}}_{k \mid k-1}^{\tau} & \mathbf{P}_{k \mid k-1}^{\tilde{\tau}}\end{array}\right],} & j=0 \\ \operatorname{EKF}_{\mathrm{U}}\left(\mathbf{z}_{k, i}^{\tilde{\tau}}, \mathbf{R}_{k}, \hat{\mathbf{x}}_{k \mid k-1}^{\tau}, \mathbf{P}_{k \mid k-1}^{\tau}, h_{k}^{s}\right), j>0,\end{cases}$

where $\mathbf{E K F _ { U }}$ is the extended Kalman filter update procedure, and the predicted measurement function is $h_{k}^{s}=$ $h_{k}^{s}\left(\hat{\mathbf{x}}_{k \mid k-1}^{\tau}, \mathbf{x}_{s}\right)$.

The posterior probability of target existence with respect to track $\tau$ at time $k$ is obtained by

$$
P\left(\chi_{k}^{\tau} \mid \mathbf{Z}^{k}\right)=\sum_{\tilde{\tau} \in \mathrm{E}(\tau)} P\left(\chi_{k}^{\tilde{\tau}} \mid \mathbf{Z}^{k}\right) / N_{s}
$$

\section{Sequential processing JIPDA (SP-JIPDA)}

The authors propose another methodology named sequential processing JIPDA (SP-JIPDA) for target tracking in clutter for this multistatic passive radar system, which avoids the association between measurements and illuminators by sequentially processing the tracks for each illuminator with all the measurements in the common measurement set, and then recursively calculates the probability of target existence as a track quality measure for track management. Note that the concept of the supertarget is not needed in this approach.

\subsection{Sequential processing framework}

The sequential implementation for multisensor multitarget tracking in clutter is introduced in [19, 20], which processes the measurements from one sensor at a time, and the measurements of the next sensor are then used to further improve the intermediate state estimation. However, the measurements of different illuminators in the multistatic passive radar system in a single frequency (DAB/DVB) network are indistinguishable, the typical sequential approach needs to be modified slightly. The sequential processing method proposed in this section is that the hybrid state of each track is updated sequentially for each illuminator with all the measurements in the common measurement set.

As shown in Fig. 1, each track state is first updated for illuminator 1 with all of the measurements of measurement set $\mathbf{Z}_{k}$ to obtain the first intermediate track state, then the intermediate track state is improved by the sequential update for the next illuminator with the common measurement set $\mathbf{Z}_{k}$. The track state update is continued till its corresponding intermediate track state is updated for the last illuminator with the common measurement set $\mathbf{Z}_{k}$. This sequential processing implementation subtly avoids the 3-D association among measurement, target and illuminator, and tangibly improves the track state estimation.

\subsection{Sequential processing JIPDA implementation}

The JIPDA $[18,28]$ is a multitarget tracking algorithm with single scan data association and the capability of track management using the probability of target existence. The sequential processing JIPDA (SP-JIPDA) algorithm is implemented by sequentially operating the JIPDA tracker to update each track $\tau$ for each illuminator with the common measurement set $\mathbf{Z}_{k}$. The proposed algorithm starts the recursion with the updated probability density function (pdf) of the track state at time $k-1$, which consists of the target existence and track trajectory state, as

$p\left(\chi_{k-1}^{\tau}, \mathbf{x}_{k-1}^{\tau} \mid \mathbf{Z}^{k-1}\right)=P\left(\chi_{k-1}^{\tau} \mid \mathbf{Z}^{k-1}\right) p\left(\mathbf{x}_{k-1}^{\tau} \mid \mathbf{Z}^{k-1}\right)$,

where

$$
p\left(\mathbf{x}_{k-1}^{\tau} \mid \mathbf{Z}^{k-1}\right)=\mathcal{N}\left(\mathbf{x}_{k-1}^{\tau} ; \hat{\mathbf{x}}_{k-1 \mid k-1}^{\tau}, \mathbf{P}_{k-1 \mid k-1}^{\tau}\right) .
$$

The track $\tau$ state at time $k$ is updated sequentially for each illuminator $s$ using the common measurement set $\mathbf{Z}_{k}$ in a recursive manner. The recursion includes

- Track state propagation

- Measurement selection and likelihood evaluation

- Multitarget data association

- Track state update

\subsubsection{Track state propagation}

The propagated track state at time $k-1$ of track $\tau$ with respect to the illuminator $s$ equals

$p\left(\chi_{k}^{\tau}(s), \mathbf{x}_{k}^{\tau}(s) \mid \mathbf{Z}^{k-1}\right)=P\left(\chi_{k}^{\tau}(s) \mid \mathbf{Z}^{k-1}\right) p\left(\mathbf{x}_{k}^{\tau}(s) \mid \mathbf{Z}^{k-1}\right)$,

where

$$
p\left(\mathbf{x}_{k}^{\tau}(s) \mid \mathbf{Z}^{k-1}\right)=\mathcal{N}\left(\mathbf{x}_{k}^{\tau}(s) ; \hat{\mathbf{x}}_{k \mid k-1}^{\tau}(s), \mathbf{P}_{k \mid k-1}^{\tau}(s)\right) .
$$

Within the sequential processing framework, the propagated track state for the first illuminator is the predicted track state $p\left(\chi_{k}^{\tau}, \mathbf{x}_{k}^{\tau} \mid \mathbf{Z}^{k-1}\right)$, and the propagated track state for the other illuminator $s$ equals the posterior track state with respect to the last illuminator $p\left(\chi_{k}^{\tau}(s-1), \mathbf{x}_{k}^{\tau}(s-\right.$ $\left.1) \mid \mathbf{Z}^{k}\right)$. The propagated probability of target existence with respect to the illuminator $s$ is obtained by

$$
P\left(\chi_{k}^{\tau}(s) \mid \mathbf{Z}^{k-1}\right)= \begin{cases}P\left(\chi_{k}^{\tau} \mid \mathbf{Z}^{k-1}\right), & s=1 \\ P\left(\chi_{k}^{\tau}(s-1) \mid \mathbf{Z}^{k}\right), & s \neq 1,\end{cases}
$$




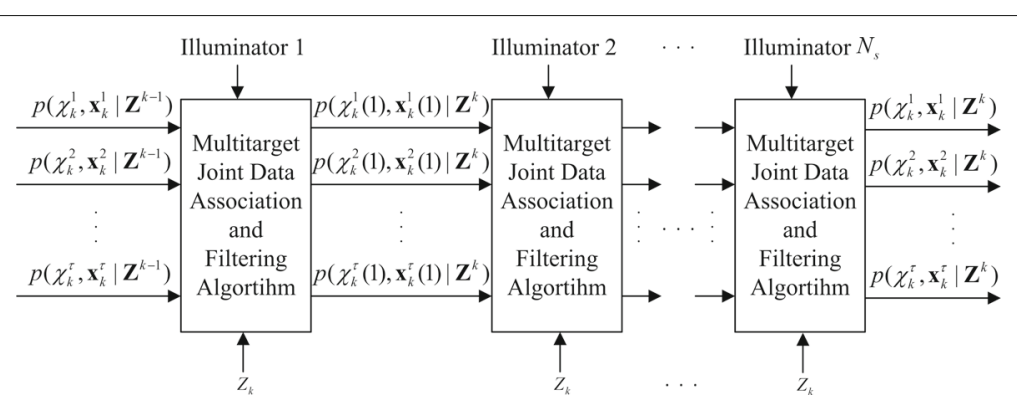

Fig. 1 Sequential processing framework

where

$$
P\left(\chi_{k}^{\tau} \mid \mathbf{Z}^{k-1}\right)=p_{1,1} P\left(\chi_{k-1}^{\tau} \mid \mathbf{Z}^{k-1}\right) .
$$

Here $p_{1,1}$ is the probability of target existence at time $k$ given that it exists at time $k-1[21]$, and $P\left(\chi_{k}^{\tau}(s-1) \mid Z^{k}\right)$ is the posterior probability of target existence with respect to illuminator $s-1$.

The propagated track trajectory state for the illuminator $s$ is given by

$\left[\hat{\mathbf{x}}_{k \mid k-1}^{\tau}(s), \mathbf{P}_{k \mid k-1}^{\tau}(s)\right]= \begin{cases}{\left[\hat{\mathbf{x}}_{k \mid k-1}^{\tau}, \mathbf{P}_{k \mid k-1}^{\tau}\right],} & s=1 \\ {\left[\hat{\mathbf{x}}_{k \mid k}^{\tau}(s-1), \mathbf{P}_{k \mid k}^{\tau}(s-1)\right],} & s \neq 1,\end{cases}$

where

$$
\left[\hat{\mathbf{x}}_{k \mid k-1}^{\tau}, \mathbf{P}_{k \mid k-1}^{\tau}\right]=\mathbf{E K F}_{P}\left(\hat{\mathbf{x}}_{k-1 \mid k-1}^{\tau}, \mathbf{P}_{k-1 \mid k-1}^{\tau}, \mathbf{F}, \mathbf{Q}_{k}\right)
$$

where $\mathbf{E K F}_{P}$ denotes the extended Kalman filter prediction procedure, and $\hat{\mathbf{x}}_{k \mid k}^{\tau}(s-1)$ and $\mathbf{P}_{k \mid k}^{\tau}(s-1)$ are the posterior mean and covariance of the track trajectory state with respect to the illuminator $s-1$ at time $k$.

\subsubsection{Measurement selection and likelihood evaluation}

For the sake of saving computational resources, the measurement selection procedure is performed to select a subset of measurements $\mathbf{z}_{k}^{\tau}(s)$ with cardinality $m_{k}^{\tau}(s)$ by each track $\tau$ with respect to each illuminator $s$.

$$
\begin{aligned}
& \mathbf{z}_{k}^{\tau}(s)= \\
& \left\{\mathbf{Z}_{k, i} \in \mathbf{Z}_{k}:\left(\mathbf{Z}_{k, i}-h_{k}^{s}\left(\mathbf{x}_{k \mid k-1}^{\tau}(s), \mathbf{x}_{s}\right)\right)^{T}\left(\mathbf{S}_{k}^{\tau}(s)\right)^{-1}\right. \\
& \left.\left(\mathbf{Z}_{k, i}-h_{k}^{s}\left(\mathbf{x}_{k \mid k-1}^{\tau}(s), \mathbf{x}_{s}\right)\right)<\kappa\right\}
\end{aligned}
$$

with

$$
\mathbf{S}_{k}^{\tau}(s)=\mathbf{H}_{k}^{\tau}(s) \mathbf{P}_{k \mid k-1}^{\tau}(s)\left(\mathbf{H}_{k}^{\tau}(s)\right)^{T}+\mathbf{R}_{k},
$$

where $\mathbf{H}_{k}^{\tau}(s)$ is the Jacobian matrix of the measurement function $h_{k}^{s}($.$) evaluated at \hat{\mathbf{x}}_{k \mid k-1}^{\tau}(s)$. Denote the $i$ th measurement of $\mathbf{z}_{k}^{\tau}(s)$ by $\mathbf{z}_{k, i}^{\tau}(s)$. Then the likelihood of measurement $\mathbf{z}_{k, i}^{\tau}(s)$ with respect to track $\tau$ and illuminator $s$ is calculated by

$$
p_{k, i}^{\tau}(s)=\frac{\mathcal{N}\left(\mathbf{z}_{k, i}^{\tau}(s) ; h_{k}^{s}\left(\hat{\mathbf{x}}_{k \mid k-1}^{\tau}(s), \mathbf{x}_{k}^{s}\right), \mathbf{S}_{k}^{\tau}(s)\right)}{P_{G}} .
$$

\subsubsection{Multitarget data association}

In multitarget tracking, as the measurement origins are no longer independent, the allocation between measurements and targets must be considered jointly or globally. As the number of feasible joint events for measurement to target allocation increases combinatorially, tracks are separated into clusters of tracks that share selected measurements. The multitarget data association operations are performed on each cluster of tracks simultaneously and independently. As the multitarget data association equations can be easily found in [28], they are interpreted by the pseudo-function of joint multitarget data association (JMTDA)

$$
\begin{aligned}
& {\left[\left\{P\left(\chi_{k}^{\tau}(s) \mid \mathbf{Z}^{k}\right),\left\{\beta_{k, i}^{\tau}(s)\right\}_{i \geq 0}\right\}_{\tau}\right]=\text { JMTDA }} \\
& {\left[\left\{P\left(\chi_{k}^{\tau}(s) \mid \mathbf{Z}^{k-1}\right),\left\{p_{k, i}^{\tau}(s)\right\}_{i>0}\right\}_{\tau}\right] .}
\end{aligned}
$$

\subsubsection{Track update}

The updated track state at time $k$ of track $\tau$ with respect to the illuminator $s$ equals

$$
p\left(\chi_{k}^{\tau}(s), \mathbf{x}_{k}^{\tau}(s) \mid \mathbf{Z}^{k}\right)=P\left(\chi_{k}^{\tau}(s) \mid \mathbf{Z}^{k}\right) p\left(\mathbf{x}_{k}^{\tau}(s) \mid \mathbf{Z}^{k}\right)
$$

where the track $\tau$ trajectory state is approximated by a single Gaussian,

$$
\begin{aligned}
p\left(\mathbf{x}_{k}^{\tau}(s) \mid \mathbf{Z}^{k}\right) & =\mathcal{N}\left(\mathbf{x}_{k}^{\tau}(s) ; \hat{\mathbf{x}}_{k \mid k}^{\tau}(s), \mathbf{P}_{k \mid k}^{\tau}(s)\right) \\
& =\sum_{i=0}^{m_{k}^{\tau}(s)} \beta_{k, i}^{\tau}(s) \mathcal{N}\left(\mathbf{x}_{k}^{\tau}(s) ; \hat{\mathbf{x}}_{k \mid k, i}^{\tau}(s), \mathbf{P}_{k \mid k, i}^{\tau}(s)\right),
\end{aligned}
$$


where the track state estimate $\left[\hat{\mathbf{x}}_{k \mid k, i}^{\tau}(s), \mathbf{P}_{k \mid k, i}^{\tau}(s)\right]$ is expressed as, when $i=0$

$$
\left[\hat{\mathbf{x}}_{k \mid k, i}^{\tau}(s), \mathbf{P}_{k \mid k, i}^{\tau}(s)\right]=\left[\mathbf{x}_{k \mid k-1}^{\tau}(s), \mathbf{P}_{k \mid k-1}^{\tau}(s)\right]
$$

and when $i>0$, as

$\left[\hat{\mathbf{x}}_{k \mid k, i}^{\tau}(s), \mathbf{P}_{k \mid k, i}^{\tau}(s)\right]=\mathbf{E K F}_{\mathbf{U}}\left(\mathbf{z}_{k, i}^{\tau}(s), \mathbf{R}_{k}, \mathbf{x}_{k \mid k-1}^{\tau}(s), \mathbf{P}_{k \mid k-1}^{\tau}(s), h_{k}^{s}\right)$

This recursive procedure with respect to each track $\tau$ at time $k$ operates for each illuminator $s=1, \ldots, N_{s}$.

\subsubsection{Track output}

The posterior track $\tau$ state at time $k$ is the track $\tau$ state updated for the last $\left(\mathcal{N}_{s}\right)$ illuminator using the common measurement set $\mathbf{Z}_{k}$,

$$
p\left(\chi_{k}^{\tau}, \mathbf{x}_{k}^{\tau} \mid \mathbf{Z}^{k}\right)=P\left(\chi_{k}^{\tau}\left(N_{s}\right) \mid \mathbf{Z}^{k}\right) p\left(\mathbf{x}_{k}^{\tau}\left(N_{s}\right) \mid \mathbf{Z}^{k}\right) .
$$

\section{Simulation validation}

In this section, the numerical experiments for multitarget tracking in a multistatic passive radar system under the $\mathrm{DAB} / \mathrm{DVB}$ network are discussed, in which the superiority of the proposed algorithm (SP-JIPDA) over the algorithm (MJIPDA) is validated and the track management in both algorithms using the probability of target existence as a track quality measure turns out to be efficient.

\subsection{Scenario description in the DAB/DVB network}

We consider a 3-D scenario of single DAB/DVB-network consisting of four illuminators and one receiver, the illuminators and receiver are all statically located on the ground ( $x-y$ plane). The multitarget geometry in this scenario is shown in Fig. 2. Five targets are simulated with a constant velocity. Targets 1,2 and 3 move in a close distance and cross each other at a small intersection angle, targets 4 and 5 start to move at the same position. All targets appear simultaneously at scan 1 except target 3 born at scan 12, and all this five targets keep surviving until the last scan (scan 40). Targets 1, 2, and 3 move towards the illuminator-receiver plane while targets 4 and 5 move apart from it.

Each pair of illuminator and receiver measure the targets independently. The probability of detection $P_{D}$ of each illuminator is modeled according the transmission loss, which depends on the distances among illuminator, target, and receiver, for simplicity, in this scenario, the detection probability $P_{D}$ is assumed equal for all targets and illuminators. In order to make the simulation challenged, the targets moving space is corrupted with heavy clutter measurements as shown in Fig. 3, where each target is measured by four pairs of illuminatorsreceiver. The maximum detection bistatic range is $14 \mathrm{~km}$, and the minimum detection bistatic range is $1 \mathrm{~km}$, as well as the maximum bistatic range rate $50 \mathrm{~m} / \mathrm{s}$ and $\mathrm{min}$ imum bistatic range rate $-50 \mathrm{~m} / \mathrm{s}$, and the number of clutter measurements follows a Poisson distribution and the clutter measurements are assumed to be uniformly distributed in this bistatic range and bistatic range rate surveillance space. $N=100$ Monte Carlo runs are performed, each run has 40 scans and each scan is sampled by $3 \mathrm{~s}$. The measurement noise follows a Gaussian distribution, $N\left(r ; 0, \sigma_{r}^{2}\right)$ for the range, and $N\left(\dot{r} ; 0, \sigma_{\dot{r}}^{2}\right)$ for the range rate.

\subsection{Performance measure criterion}

Due to the association ambiguity between measurements and illuminators as well as the unavailability of angle information, the track initiation using measurements in the multistatic passive radar with the DAB/DVB network is significantly computationally expensive and usually fails to give satisfactory performance, thus, is outside the scope of this paper; preliminary work can be found in [29], wherein the track initiation performance is quite sensitive to the clutter and target detection probability, and works only with very small number of clutter measurements and high target detection probability.

In this simulation, the target ground truth information is used to initiate a track [15]. The initiated track position and velocity are generated from the target truth position and velocity with a certain disturbance (assumed to be a Gaussian distribution), i.e., the initiation position of target $\tau$ is $\hat{\mathbf{x}}_{0}^{\tau, p}=\mathbf{x}_{0}^{\tau, p}+N\left(p_{0} ; 0, \sigma_{I}^{2}\right)$, and the initiation velocity is $\hat{\mathbf{x}}_{0}^{\tau, \nu}=\mathbf{x}_{0}^{\tau, \nu}+N\left(v_{0} ; 0,10^{-4} \sigma_{I}^{2}\right)$, where $\mathbf{x}_{0}^{\tau, p}$ and $\mathbf{x}_{0}^{\tau, \nu}$ are the true initial target position and velocity of target $\tau$, respectively.

\subsubsection{Track management}

An important performance measure for target tracking in clutter is the track management. In this simulation, both the MJIPDA and the SP-JIPDA algorithms calculate the probability of target existence as the track quality measure for track management. Each initiated track is given an initial value as the probability of target existence, which is recursively updated by the measurements in the subsequent scans. The track management procedure confirms a track if its updated probability of target existence is greater than a predefined confirmation threshold, and it maintains the confirmed status until terminated when the updated probability of target existence falls below a predefined termination threshold.

Since the tracks are initialized based on the targets' ground truth information at targets' born time, only the tracks following targets are initialized. In this experiments, the average number of confirmed tracks (ANCT) 


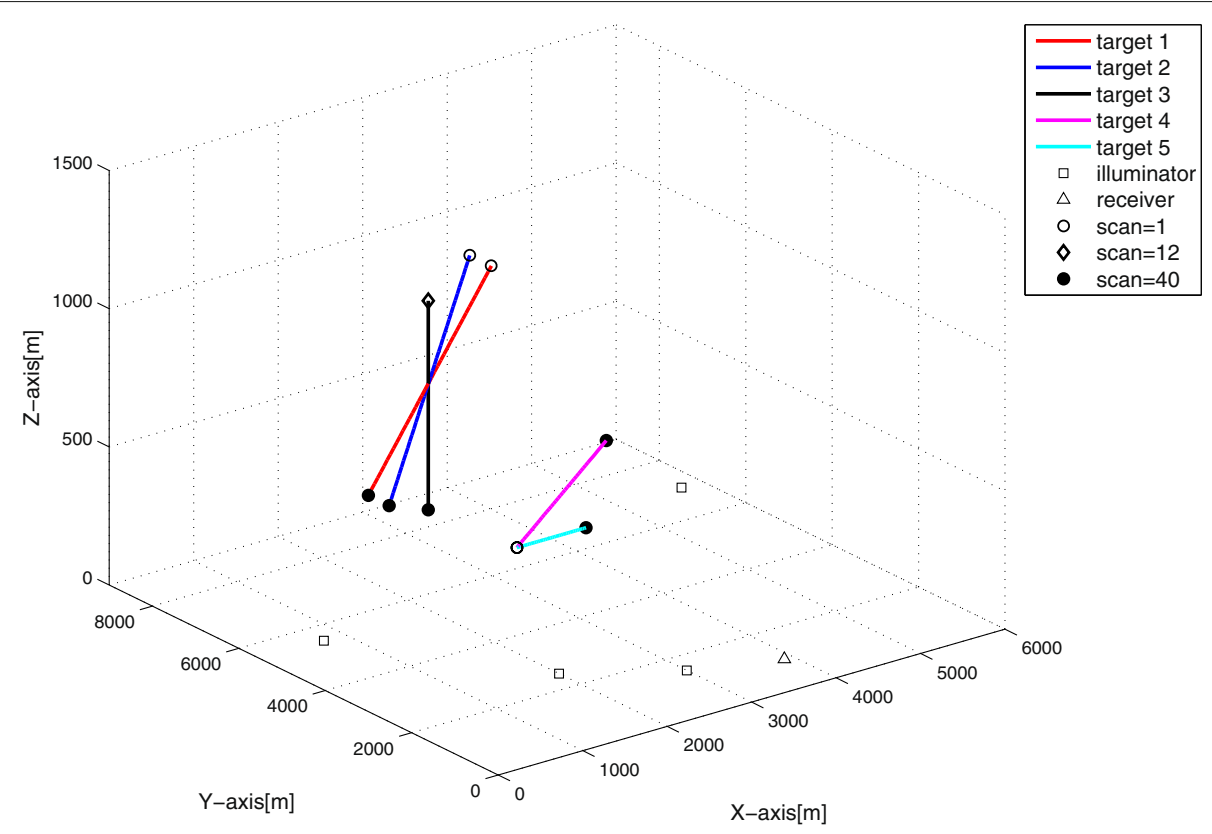

Fig. 2 Targets geometry in a DAB/DVB network

is utilized as the track management measure. It is averaged over $N$ Monte Carlo runs and can be obtained by

$$
\operatorname{ANCT}(N)=\frac{1}{N} \sum_{i=1}^{N} N_{c}^{i}
$$

where $N_{c}^{i}$ is the number of confirmed tracks at $i$ th Monte Carlo run. For fair performance comparison of track maintenance, both SP-JIPDA and MJIPDA algorithms are given the same track management parameters (the initial probability of target existence, the track confirmation threshold and termination threshold).

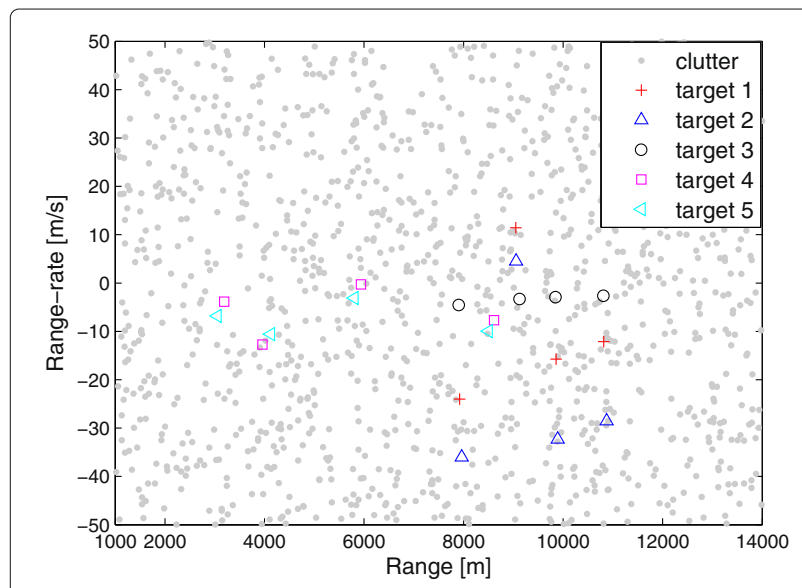

Fig. 3 Example of received measurements for one scan with clutter density $\rho=0.001$

\subsubsection{Trajectory average estimation error}

The root mean square error (RMSE) is an efficient trajectory average estimation error. It is averaged over all $N$ Monte Carlo runs and can be calculated by

$$
\operatorname{RMSE}(k)=\sqrt{\frac{1}{N} \sum_{i=1}^{N}\left\|\mathbf{x}_{k}^{\tau}-\mathbf{x}\right\|}
$$

where $\mathbf{x}_{k}^{\tau}$ is the trajectory estimation of track $\tau$ at time $k$ and $\mathbf{x}$ is the true target state.

\subsection{Numerical results}

In order to verify the robustness of the proposed algorithm, two cases of experiments with respect to different environment parameters are simulated as follows:

- Case 1: detection probability $P_{D}=0.9$, measurement noise deviation $\sigma_{r}=10 \mathrm{~m}$ and $\sigma_{\dot{r}}=0.1 \mathrm{~m} / \mathrm{s}$, clutter measurement density $\rho=0.001$ (number $/ \mathrm{m}^{2} . s^{-1}$ ), Gaussian disturbance deviation $\sigma_{I}=10 \mathrm{~m}$.

- Case 2: detection probability $P_{D}=0.9$, measurement noise deviation $\sigma_{r}=30 \mathrm{~m}$ and $\sigma_{\dot{r}}=0.3 \mathrm{~m} / \mathrm{s}$, clutter measurement density $\rho=0.001$ (number $/ \mathrm{m}^{2} . s^{-1}$ ), Gaussian disturbance deviation $\sigma_{I}=40 \mathrm{~m}$.

The track management performance across the proposed algorithms is compared using the average number of confirmed tracks and is shown in Fig. 4. As can be seen, in both cases, the proposed two algorithms succeed to confirm all the targets after several scans' delay, which firmly validates the probability of target existence 


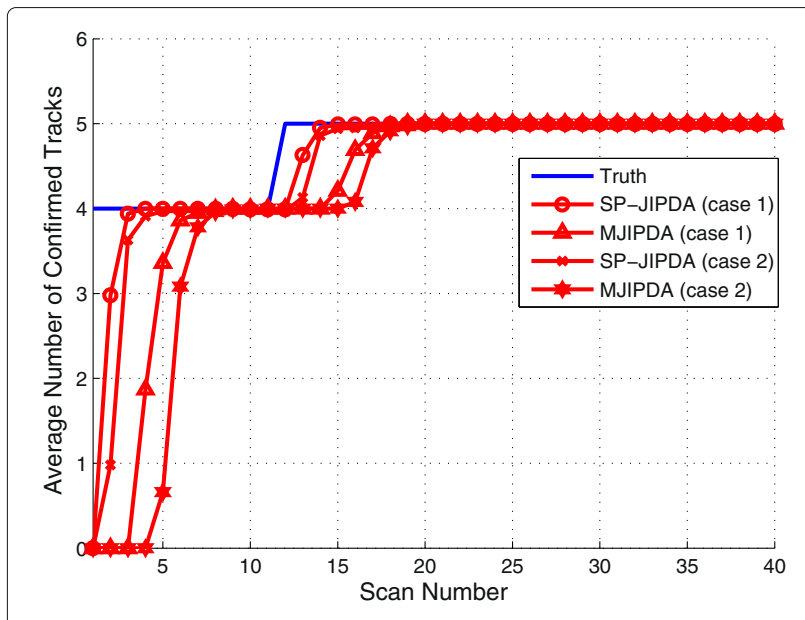

Fig. 4 Average number of confirmed tracks

that recursively calculated in both algorithms is an efficient track quality measure for track management. What is more, in each case, the proposed SP-JIPDA algorithm always delivers improved track management performance over the MJIPDA algorithm under the given simulation scenario, i.e., the SP-JIPDA algorithm confirms the targets earlier and faster than the MJIPDA algorithm. In case 1, the SP-JIPDA starts to confirm tracks at scan 2 and thereafter takes 4 scans' time to completely confirm four targets; however, the MJIPDA starts to confirm tracks at scan 4 and needs 8 scans' time to confirm all four targets, besides, when the fifth target appears at scan 12, the SP-JIPDA algorithm only takes 4 scans' time to confirm it rather than 9 scans' time needed by the MJIPDA. In case 2 , the track management performances of both the SPJIPDA and MJIPDA algorithm degenerate slightly due to the increased measurement noise and larger initialization error compared with that of in case 1, but the SP-JIPDA

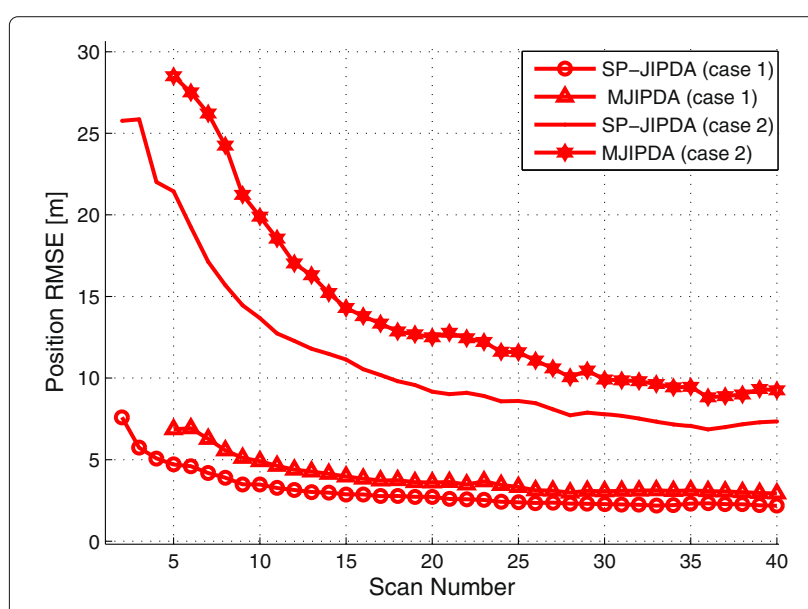

Fig. 5 RMSE of target 1

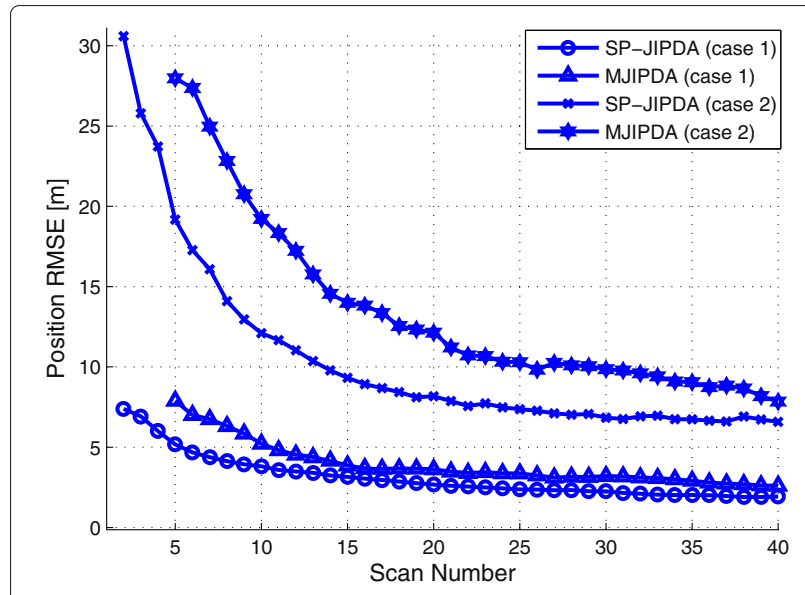

Fig. 6 RMSE of target 2

algorithm still confirms the targets earlier and faster over the MJIPDA algorithm.

Meanwhile, the position RMSEs of the confirmed tracks that follows targets are presented in Figs. 5, 6, 7, 8, and 9, respectively. As can be found therein, the SP-JIPDA algorithm performs significantly better than the MJIPDA algorithm in both cases 1 and 2 . In case 1, the position RMSE of the SP-JIPDA with respect to each target is about 1 $m$ less than that of the MJIPDA. In case 2, the position RMSEs of both algorithms increase significantly due to the increased measurement noise and initialization errors, but the SP-JIPDA algorithm still outperforms the MJIPDA with over $2 \mathrm{~m}$ position estimation error less.

As a consequence, the SP-JIPDA algorithm delivers much better tracking performance in terms of both track management and trajectory estimation. This can be explained by that the track state at each scan in SPJIPDA algorithm is improved multiple times via sequential

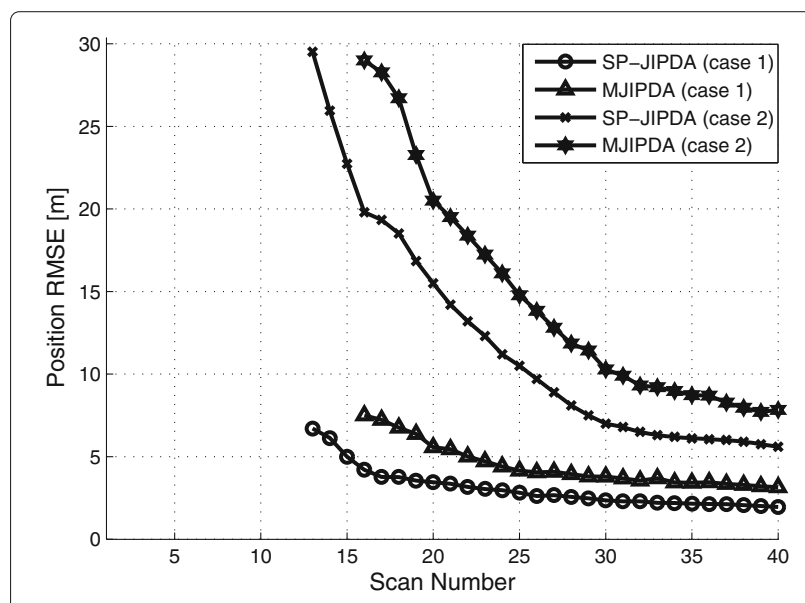

Fig. 7 RMSE of target 3 


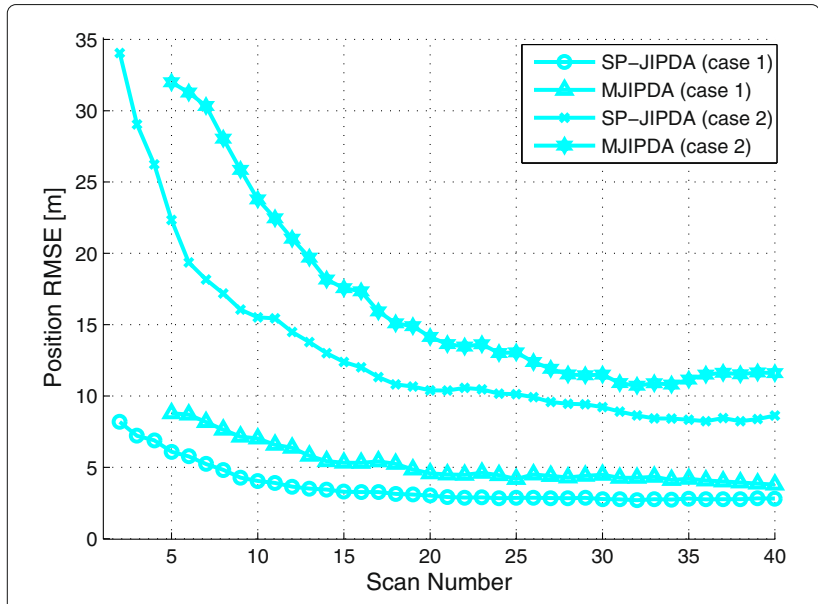

Fig. 8 RMSE of target 4

updating with respect to various illuminators, wherein the target measurements detected by different pair of illuminator-receivers are updated by incrementally accurate predicted track states at single scan; therefore, the likelihood of target measurements obtained by the SPJIPDA algorithm is higher than that by the MJIPDA algorithm, which gives a faster increasing rate of the probability of target existence.

The execution time in experiment 1 is given in Table 2 on a PC with Intel(R) Core(TM) i7-6700 CPU 3.4 GHz and RAM 16 GB running windows 7 and Matlab R2013a program. As shown in Table 2, the SP-JIPDA algorithm requires less computation time compared with the MJIPDA algorithm, and both algorithms are capable of real-time application since their execution times are much smaller than the entire simulation time.

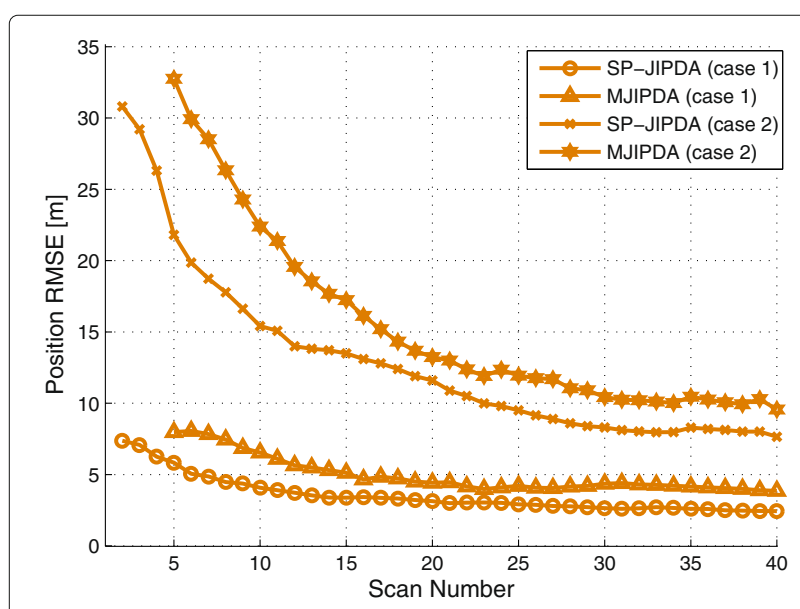

Fig. 9 RMSE of target 5
Table 2 Execution time [sec.]

\begin{tabular}{lll}
\hline & Case 1 & Case 2 \\
\hline MJIPDA & 750 & 832 \\
SP-JIPDA & 620 & 698 \\
Entire simulation time & 12,000 & 12,000 \\
\hline
\end{tabular}

\section{Conclusions}

This paper investigates two solutions for multitarget tracking in clutter directly in 3-D Cartesian coordinates using multistatic passive radar with a DAB/DVB network, where both algorithms are capable of track management using the probability of target existence as the track quality measure.

The MJIPDA algorithm is developed by incorporating the probability of target existence into the MJPDA algorithm as a track quality measure for track management, and the MJPDA algorithm addresses the target-measurement illuminator association ambiguity via "supertargets" using gate grouping. The SP-JIPDA algorithm sequentially operates the JIPDA tracker to update each track for each illuminator with the common measurement set at each scan. Compared with the MJIPDA algorithm, the SP-JIPDA enhances the target's track multiple times only at single scan by sequentially processing with respect to various illuminators; therefore, the target measurements can be utilized in a more efficient way to update the target's track state. The simulation validates the efficiency of the proposed algorithm and also shows the superiority performance of SP-JIPDA over the MJIPDA algorithm.

There have several aspects worthy of further work: the availability and robustness of the proposed algorithms are worthwhile to be validated based on the real data obtained from a realistic setup of multistatic passive radar system under the DAB/DVB network; the more computationally efficient versions of the proposed multitarget tracking algorithm are attractive to be developed since the proposed multitarget tracking algorithms employ the optimal Bayesian multitarget joint data association approach which may suffer from the numerical explosion when numbers of close targets presented in the surveillance space; the extension of the proposed algorithms to multiple maneuver targets tracking in cluttered environment is also valuable.

\section{Nomenclature}

\section{A. List of acronyms}

DAB/DVB Digital audio/video broadcast

JPDA Joint probabilistic data association

JIPDA Joint integrated probabilistic data association

MJIPDA Modified joint integrated probabilistic data association 
SP-JIPDA Sequential processing-joint integrated probabilistic data association

TDOA Time difference of arrival

EKF Extended Kalman filter

UKF Unscented Kalman filter

BPF Bootstrap particle filter

APF Auxiliary particle filter

TBD Track-before-detect

PMHT Probabilistic multi-hypothesis tracker

FJE Feasible joint event

JMTDA Joint multitarget data association

ANCT Average number of confirmed tracks

RMSE Root mean square error

\section{B. List of symbols}

$\|\mathbf{x}\|$ The euclidean norm of vector $\mathbf{x}$

$\mathbf{x}^{T}$ The transpose of vector $\mathbf{x}$

$\otimes$ The Kronecker product

$\mathbf{I}_{3}$ The identity matrix of size 3

$s$ A illuminator indexed by $s$

$\tau$ A track or a target followed by certain track by superscript $\tau$

$\tilde{\tau}$ A hypothetical target consisting of a pair of target $\tau$ and illuminator $s$

$\chi_{k}^{\tau}$ Event that target $\tau$ exists at time $k$

$\chi_{k}^{\tau}(s)$ Event that target $\tau$ exists at time $k$ for illuminator $s$

$\mathbf{x}_{k}^{\tau}$ The trajectory state in 3-D Cartesian coordinates of target $\tau$ at time $k$

$\mathbf{x}_{k}^{\tau, p}$ The position component of $\mathbf{x}_{k}^{\tau}$

$\mathbf{x}_{k}^{\tau, v}$ The velocity component of $\mathbf{x}_{k}^{\tau}$

$\mathbf{x}_{k}^{\tau}(s)$ Trajectory state in 3-D Cartesian coordinates of target $\tau$ at time $k$ for illuminator $s$

$\mathbf{x}_{s}$ The position of illuminator $s$ in 3-D Cartesian coordinates

$\mathbf{x}_{r}$ The position of the only receiver in 3-D Cartesian coordinates

$N_{s}$ The number of entire illuminators

$\gamma_{k}$ Sensor received bistatic range measurement at time $k$

$\dot{\gamma}_{k}$ Sensor received bistatic range-rate measurement at time $k$

F Target trajectory transition matrix

$\mathbf{Q}_{k}$ Target trajectory plant noise covariance matrix

$\mathbf{R}_{k} \quad$ Measurement noise covariance matrix

$P_{D}$ Target detection probability

$P_{G}$ Gating probability that the (true) measurement will fall in the gate

$\mathbf{Z}_{k}$ Sensor received set of measurements at time $k$

$\mathbf{Z}_{k, i}$ The ith measurement of $\mathbf{Z}_{k}$
$\mathbf{Z}^{k} \quad$ Set of measurements up to and including time $k$

$\rho_{k, i}$ Clutter measurement density of $\mathbf{Z}_{k, i}$

$\mathbf{z}_{k}^{\tilde{\tau}}$ Set of selected measurements at time $k$ with respect to supertarget $\tilde{\tau}$

$N_{k}^{\tilde{\tau}} \quad$ The cardinality of $\mathbf{z}_{k}^{\tilde{\tau}}$

$\mathbf{z}_{k, i}^{\tilde{\tau}}$ The $i$ th measurement of $\mathbf{z}_{k}^{\tilde{\tau}}$

$\xi_{j}$ The $j$ th feasible joint event

$\mathbf{z}_{k}^{\tau}(s)$ Set of selected measurements at time $k$ with respect to track $\tau$ for illuminator $s$

$m_{k}^{\tau}(s)$ The cardinality of $\mathbf{z}_{k}^{\tau}(s)$

$\mathbf{z}_{k, i}^{\tau}(s)$ The ith measurement of $\mathbf{z}_{k}^{\tau}(s)$

$T_{0}\left(\xi_{j}\right)$ The set of supertargets allocated no measurement in $\xi_{j}$

$T_{1}\left(\xi_{j}\right)$ The set of supertargets allocated one measurement in $\xi_{j}$

$\Xi(\tilde{\tau}, i)$ The set of FJEs which allocate measurement $i$ to supertarget $\tilde{\tau}$

$\chi_{k, i}^{\tilde{\tau}} \quad$ Event that the selected measurement $i$ is the detection of supertarget $\tilde{\tau}$ at time $k$

$\beta_{i \tilde{\tau}}$ Posterior association probability measurement $i$ is supertarget $\tilde{\tau}$ detection

$\beta_{k, i}^{\tau}(s)$ Posterior association probability measurement $i$ is target $\tau$ detection for $s$

$E(\tau)$ The set of supertargets originated from track $\tau$

$p_{1,1}$ The probability of target existence at time $k$ given that it exists at time $k-1$

$T$ The sampling interval between two consecutive scans

$P_{f a}$ The probability of false alarm

$V_{r c}$ The sensor resolution cell volume

\section{Acknowledgements}

This work was supported by the Agency for Defense Development, Republic of Korea (Grant UD160001DD).

\section{Authors' contributions}

YS made the main contributions to conception and tracking algorithms' design, as well as drafting the article. SP mainly designed the simulation validation and results analysis. TS offered critical suggestions on the algorithms' design, provided significant revising for important intellectual content and gave final approval of the current version to be submitted. All authors read and approved the final manuscript.

\section{Competing interests}

The authors declare that they have no competing interests.

Received: 25 September 2016 Accepted: 13 January 2017

Published online: 23 January 2017

\section{References}

1. H Kuschel, J Heckenbach, S Muller, R Appel, in Radar Conference. On the potentials of passive multistatic low frequency radars to counter stealth and detect low flying targets (IEEE, Rome, 2008)

2. U Reimers, Digitale Fernsehtechnik. (Springer, Berlin, 1995)

3. H Kuschel, VHF/UHF radar. 1. characteristics. Electron. Commun. Eng. J. 14(2), 61-72 (2002)

4. PE Howland, Target tracking using television-based bistatic radar. IEE Proceedings-Radar, Sonar Navig. 146(3), 166-174 (1999) 
5. CR Berger, B Demissie, J Heckenbach, P Willett, SL Zhou, Signal processing for passive radar using OFDM waveforms. IEEE J. Sel. Top. Sign. Process. 4(1), 226-238 (2010)

6. MH Cai, F He, LN Wu, in Image and Signal Processing. Application of UKF algorithm for target tracking in DTV-based passive radar (IEEE, Tianjin, 2009), pp. 1-4

7. XF Yin, T Pedersen, P Blattnig, A Jaquier, BH Fleury, in 13th Digital Signal Processing Workshop and 5th IEEE Signal Processing Education Workshop (DSP/SPE). A single-stage target tracking algorithm for multistatic DVB-T passive radar systems (IEEE, Florida, 2009), pp. 518-523

8. D Orlando, L Venturino, M Lops, G Ricci, Track-before-detect strategies for STAP radars. IEEE Trans. Signal Process. 58(2), 933-938 (2010)

9. D Orlando, G Ricci, Y Bar-Shalom, Track-before-detect algorithms for targets with kinematic constraints. IEEE Trans. Aerosp. Electron. Syst. 47(3), 1837-1849 (2011)

10. F Ehlers, D Orlando, G Ricci, Batch tracking algorithm for multistatic sonars. IET Radar, Sonar. Navig. 6(8), 746-752 (2012)

11. RTharmarasa, N Nandakumaran, M McDonald, T Kirubarajan, in SPIE Optical Engineering + Applications. On the potentials of passive multistatic low frequency radars to counter stealth and detect low flying targets, (2009)

12. M Daun, W Koch, in Radar Conference, 2008. RADAR '08. IEEE. Multistatic target tracking for non-cooperative illumination by DAB/DVB-T (IEEE, 2008), pp. 1-6

13. D Martina, N Ulrich, W Koch, Tracking in multistatic passive radar systems using DAB/DVB-T illumination. Signal Process. 92(6), 1365-1386 (2012)

14. S Choi, CR Berger, DF Crouse, P Willett, SL Zhou, in Optical Engineering+ Applications. Target tracking for multistatic radar with transmitter uncertainty (SPIE, California, 2009)

15. S Choi, DF Crouse, P Willett, SL Zhou, Approaches to Cartesian data association passive radar tracking in a DAB/DVB network. IEEE Trans. Aerosp. Electron. Syst. 50(1), 649-663 (2014)

16. B Ristic, S Arulampalam, N Gordon, Beyond the Kalman filter: Particle filters for tracking applications. (Artech House, London, 2004)

17. C Hue, JP Cadre, P Pérez, Tracking multiple objects with particle filtering IEEE Trans. Aerosp. Electron. Syst. 38(3), 791-812 (2002)

18. D Mušicki, R Evans, Joint Integrated Probabilistic Data Association - JIPDA. IEEE Trans. Aerosp. Electron. Syst. 40(3), 1093-1099 (2004)

19. Y Bar-Shalom, T Fortman, Tracking and Data Association. (Academic Press, Cambridge, 1988)

20. LY Pao, CW Frei, in Proceedings of the 1995 American Control Conference. A comparison of parallel and sequential implementations of a multisensor multitarget tracking algorithm (IEEE, Seattle, 1995), pp. 1683-1687

21. D Mušicki, R Evans, S Stanković, Integrated Probabilistic Data Association (IPDA). IEEE Trans. Autom. Control. 39(6), 1237-1241 (1994)

22. U Khan, YF Shi, TL Song, Fixed lag smoothing target tracking in clutter for a high pulse repetition frequency radar. EURASIP J. Adv. Signal Process. 2015(1), 1-11 (2015)

23. D Mušicki, BL Scala, R Evans, The integrated track splitting filter-Effcient multi-scan single target tracking. IEEE Trans. Aerosp. Electron. Syst. 43(4), 1405-1429 (2007)

24. Y Bar-Shalom, P Willett, X Tian, Tracking and Data Association:A Handbook of Algorithms. (YBS Publishing, Storrs, 2011)

25. T Hanselmann, D Mušicki, in 2005 7th International Conference on Information Fusion. Optimal signal detection for false track discrimination, (2005)

26. TL Song, D Mušicki, Adaptive Clutter Measurement Density Estimation for Improved Target Tracking. IEEE Trans. Aerosp. Electron. Syst. 47(2), 1457-1466 (2011)

27. DF Crouse, Y Bar-Shalom, P Willett, L Svensson, in Defense, Security, and Sensing. The JPDAF in practical systems: Computation and snake oil (SPIE, Orlando, 2010)

28. SChalla, R Evans, M Morelande, Fundamentals of Object Tracking. (Cambridge University Press, Cambridge, 2011)

29. S Choi, DF Crouse, P Willett, SL Zhou, Multistatic target tracking for passive radar in a DAB/DVB network: initiation. IEEE Trans. Aerosp. Electron. Syst. 51(3), 2460-2469 (2015)

\section{Submit your manuscript to a SpringerOpen ${ }^{\circ}$ journal and benefit from:}

- Convenient online submission

- Rigorous peer review

- Immediate publication on acceptance

- Open access: articles freely available online

- High visibility within the field

- Retaining the copyright to your article

Submit your next manuscript at $\gg$ springeropen.com 